\title{
Serial Assessment of Circulating T lymphocyte Phenotype and Receptor Repertoire During Treatment of Non-Muscle Invasive Bladder Cancer with Adoptive T Cell Immunotherapy
}

\section{Xiaoli Wang}

Capital Medical University Affiliated Beijing Shijitan Hospital

\section{Guoliang Qiao}

Capital Medical University Affiliated Beijing Shijitan Hospital

Ni Jiang

Capital Medical University Affiliated Beijing Shijitan Hospital

Michael A Morse

Duke University Medical Center

Xinna Zhou

Capital Medical University Affiliated Beijing Shijitan Hospital

\section{Shuo Wang}

Capital Medical University Affiliated Beijing Shijitan Hospital

\section{Jiangping Wu}

Capital Medical University Affiliated Beijing Shijitan Hospital

\section{Yuguang Song}

Capital Medical University Affiliated Beijing Shijitan Hospital

\section{Yanjie Zhao}

Capital Medical University Affiliated Beijing Shijitan Hospital

\section{Lei Zhou}

Capital Medical University Affiliated Beijing Shijitan Hospital

\section{Yanhua Yuan}

Capital Medical University Affiliated Beijing Shijitan Hospital

\section{Amy Hobeika}

Duke University Medical Center

Jun Ren ( $\nabla$ jun.ren@duke.edu )

Capital Medical University Affiliated Beijing Shijitan Hospital https://orcid.org/0000-0001-5399-000X

Herbert Kim Lyerly

Duke University Medical Center

\section{Research}

Keywords: Adoptive T cell immunotherapy, bladder cancer, $\mathrm{T}$ cell receptor repertoire

Posted Date: September 23rd, 2020

DOI: https://doi.org/10.21203/rs.3.rs-79476/v1

License: (c) (i) This work is licensed under a Creative Commons Attribution 4.0 International License. Read Full License 


\section{Abstract}

Background: Recurrence and progression of non-muscle-invasive bladder cancer (NMIBC) are frequent despite intravesical therapy. We hypothesized that administration of an activated T cell immunotherapy (ACT) at times when immunosuppressive populations were noted to have increased in peripheral blood would prevent recurrence of disease.

Method: This was an N-of-1 study. A subject with multiple primary bladder high grade urothelial carcinomas who had initially completed standard resection and chemotherapy was enrolled. ACT consisted of dendritic cells mixed with cytokine induced killer cells $(\mathrm{DC} / \mathrm{CIK})$ ) which were infused intravenously 18 times over a 6 year period in response to observed increases in peripheral blood immunosuppressive $\mathrm{CD}^{+} / \mathrm{CD} 28^{-}$cells. Peripheral blood was analyzed for T cell phenotype by flow cytometry, T cell receptor (TCR) repertoire, and circulating tumor DNA (ctDNA) by next generation sequencing (NGS) at the time of each infusion. Standard of care cystoscopy and pelvic CT scans have been performed at routine intervals.

Results: The patient's resected bladder tumors did not express PD-1 or PD-L1. There has been no recurrence of urothelial carcinoma. Peripheral blood cytotoxic T cells and unique TCR clones increased and suppressive T cell populations decreased after DC/CIK infusions. ctDNA detected mutations in six tumor relevant genes (ARID1B, MYCN, CDH23, SETD2, NOTCH4 and FAT1) at baseline but all of them disappeared after the DC-CIK infusions.

Conclusion: DC/CIK infusions were associated with beneficial changes in T cell phenotype, TCR repertoire, decreases in circulating tumor DNA and sustained recurrence-free survival.

\section{Introduction}

Non-muscle-invasive transitional cell bladder cancer (NMIBC), accounting for more than $60 \%$ of bladder cancers[1], has a high recurrence rate (50\% for high grade T1 disease[2]) after transurethral resection (TURBT), despite intravesical chemotherapy or immunotherapy with bacillus Calmette-Guerin (BCG)[3]. Immune checkpoint inhibitors have demonstrated efficacy in metastatic bladder cancers and preliminary data from a study of checkpoint blockade prior to cystectomy for muscle invasive bladder cancer has demonstrated a complete response rate of 39\%[4] and studies for non-muscle invasive bladder cancer unresponsive to BCG are ongoing[5]. However, the majority of bladder cancers do not respond to those agents in part due to a "non-inflamed" tumor microenvironment [6].

A strategy to promote an inflamed tumor microenvironment is autologous adoptive T cell immunotherapy, delivery of ex vivo activated $T$ cell products. A common form of adoptive $T$ cell immunotherapy consists of a mixture of dendritic cells (DC) and cytokine induced killer (CIK) cells. Advantages of DC-CIK are feasibility of generation, rapid expansion ex vivo, and non-MHC restricted cytotoxicity $[7,8]$. We have previously demonstrated that the combination of DC-CIK infusions and S-1 chemotherapy was safe and resulted in favorable PFS and OS in both advanced pancreatic and gastric cancers $[9,10]$. Quantitively measurements of the peripheral blood TCR repertoire using high-throughput T cell receptor V $\beta$ sequencing (HTTCR)[11-13] has led to the observation that clinical responders have a greater number of expanded, unique, rather than shared, clones in the peripheral blood.

In this report, we describe a patient with multiple lesions of resected NMIBC, devoid of expression of PD-1 or PD-L1 who received standard intravesical treatment and intravenous chemotherapy followed by DC-CIK immunotherapy for 18 cycles over 63 months. During this time period, peripheral blood samples $(n=21)$ collected at each cycle of T cell immunotherapy were analyzed for circulating tumor DNA (ctDNA) mutations, TCR repertoire and T lymphocyte phenotype. Serial cross sectional imaging and cystoscopy have continued to demonstrate no evidence of recurrence.

\section{Materials And Methods}

\section{Patient}


The subject was a 62-year-old man who presented to the hospital on June 2013 with a one-month history of hematuria. TURBT revealed multiple lesions measuring $0.6 \times 0.5 \times 0.5 \mathrm{~cm}, 0.7 \times 0.5 \times 0.5 \mathrm{~cm}$, and $1.0 \times 0.5 \times 0.5 \mathrm{~cm}$. Pathological analysis demonstrated high-grade NMIBC, $\mathrm{T}_{1} \mathrm{~N}_{0} \mathrm{M}_{0}$. The patient received the institutional standard 2-weekly pirarubicin and 6weekly thiotepa intravesical instillations, and 3 monthly hydroxycamptothecin instillations concurrently with gemcitabine plus cisplatin intravenous chemotherapy for 5 cycles, each at 21-day intervals. Comparing the PET/CT from August 8, 2020 with that performed on June 8,2013 , we noted metabolic activity of the bladder consistent with early progression of disease. The subject declined radical cystectomy and subsequently enrolled into this study after providing written informed consent.

\section{Study design and procedures}

This was an N-of-1 study, performed in accordance with the Declaration of Helsinki after receiving approval from the Institutional Review Board of Beijing Shijitan Hospital. The DC-CIK cell product was prepared as described in our previous studies $[10,14]$. For the induction of DC-CIKs, peripheral blood mononuclear cells were mobilized by G-CSF. Apheresis was performed using the COBE Spectra cell separator (COBE BCT, Lakewood, CO, USA) until CD34 ${ }^{+}$cells reached $\geq 4.5 \times 10^{6} / \mathrm{kg}$. A portion of the apheresis product $(25-50 \mathrm{ml})$ was co-cultured with IL-4, TNF-a and GM-CSF in vitro to generate autologous DCs. PBMCs were activated in vitro with IL-2, INF-gamma and anti-CD3 antibody to generate the CIK cells. After meeting lot release criteria, the cultured cells were infused intravenously over 20 minutes.

Beginning in the first month post-TURBT, the ex vivo expanded DC-CIK cells were administered intravenously (median infusion of $1.95 \times 10^{9} \mathrm{ClK}$ cells, consisting of $\sim 75 \% \mathrm{CD} 8^{+} \mathrm{T}$ cells) for a total of 18 infusions over a 63 month period. Blood samples were collected for TCR repertoire and T cell phenotypic analysis and ctDNA mutational analysis during this time period. Peripheral blood $\mathrm{CD} 8^{+} / \mathrm{CD} 28^{-} \mathrm{T}$ cell levels were used as predictors for determining the timing of adoptive $\mathrm{T}$ cell infusion. We chose $24.2 \%$ of $\mathrm{CD}^{+} / \mathrm{CD}^{2} 8^{-}$as the threshold to determine the timing of DC-CIK infusions.

\section{ctDNA mutational analysis}

Next generation sequencing was performed on peripheral blood ctDNA by a commercial vendor (Geneplus-Beijing Institute, Beijing). Targeted sequencing was performed in 60 plasma ctDNA and 30 germ line DNA samples. The target region is about 1.1 Mb, which includes coding exons and selected introns of 1021 genes selected from four sources: 1) known oncogenes and tumor suppressor genes; 2) genes that are targets of agents approved by the FDA or have been assessed in clinical trials; 3) genes implicated in major cancer-related signaling pathways; and, 4) genes identified in the findings of the TCGA network which covers 12 cancer types. Sequencing libraries were prepared from ctDNA using KAPA DNA Library Preparation Kits (Kapa Biosystems, Inc.), and gDNA sequencing libraries were prepared using the protocols recommended by the Illumina TruSeq DNA Library Preparation Kit. For samples close to the minimum input requirement, additional pre-capture PCR cycles were performed to generate sufficient PCR product for hybridization. Libraries were hybridized to custom-designed biotinylated oligonucleotide probes (Integrated DNA Technology, Coralville, USA) covering the target region sequence. DNA sequencing was carried out with the HiSeq3000 Sequencing System (Illumina, San Diego, CA).

Somatic SNVs and InDels were detected using the Mutect 2.0 algorithm

(https://software.broadinstitute.org/gatk/gatkdocs/current/org_broadinstitute_gatk_tools_walkers_cancer_m2_MuTect2.php) Somatic copy number alterations and structure variations were analyzed using local algorithms.

\section{T cell receptor (TCR) sequencing of peripheral blood T cells}

DNA was extracted from peripheral blood T cells using a Qiagen DNA FFPE kit, DNA blood kit, or DNA blood mini kit (Qiagen). TCR V $\beta$ CDR3 sequencing was performed using the deep (Peripheral blood mononuclear cell, PBMC) resolution Immunoseq platforms. Bio-informatic and bio-statistical analyses of productive clones were performed to assess the dynamics of $T$ cells. The Shannon diversity index [15], TCR clonality[16] and Evenness [17] of T cells were used to evaluate the diversity of TCR CDR3 V-beta sequences.

\section{Phenotypic analysis of peripheral blood immune cells}


Whole blood $(100 \mu \mathrm{l})$ was incubated in the dark with primary antibody at $4{ }^{\circ} \mathrm{C}$ for $15 \mathrm{~min}$. Anti-CD3-FITC/anti-CD 56-RPE (Dako), anti-CD3-FITC (fluorescein isothiocyanate), anti-CD4-RPE, anti-CD8-RPE, anti-CD45RO, and anti-CD4-FITC/anti-CD25PE (BD Bioscience) were used. After hemolysis for 10 minutes, samples were centrifuged for 10 min at 1,500 rpm at room temperature, and then washed twice in PBS and subjected to three-color flow cytometry to determine cell phenotypes using an FC500 (Beckman-Coulter), and CXP analysis software (Beckman-Coulter). Lymphocytes were identified on scattergrams as the low forward scatter/low side scatter population. Analysis was performed on $\geq 5,000$ gated events.

\section{Clinical outcomes}

At the time of each cycle of cell therapy, standard physical exam, pelvic CT and cystoscopy were performed to assess the status of the tumor.

\section{Statistical analyses}

Continuous variables were expressed as mean \pm SD (standard deviation) and compared using a two-tailed unpaired Student's $t$ test. Categorical variables were compared using $\chi 2$ or Fisher analysis. All statistical evaluations were carried out using SPSS software (Statistical Package for the Social Science, version 15.0, SPSS Inc, Chicago, IL) and GraphPad Prism 5 (Version 5.01, GraphPad Software, Inc., USA). A value of $p<0.05$ was considered to be statistically significant in all the analyses.

\section{Results}

\section{Clinical Evaluation}

During 72 months of follow-up, pelvic CT and cystoscopy have demonstrated an ongoing, complete remission (Fig. 1A-F). Pathological examination of tumor tissue prior to therapy demonstrated no PD-1 or PD-L1 expressing cells Fig. 1G, H), indicating a non-inflamed tumor microenvironment unlikely to respond to immune checkpoint inhibitor treatment.

\section{Variable TCR diversity is associated with the infused T cell number}

In order to analyze the changing TCR diversity of T cells associated with the DC-CIK infusions, we measured the changing trends of Shannon index, clonality and evenness (Fig. 2A) and compared these data with the recorded number of T cells infused over the course of the study (Fig. 2B). The number of infused cells was significantly associated with the Shannon index (Fig. $2 C, r^{2}=0.295, P=0.019$ ) but not with clonality (Fig. $2 D, r^{2}=0.183, P=0.076$ ) or evenness (Fig. $2 D, r^{2}=0.130, P=$ 0.142). Considering the differences observed in $T$ cell frequency and clonality, we further investigated the overlap in $T$ cell clones of this patient. As shown in Fig. 2E, the unique TCR clones and the shared TCR clones changed after the administration of ACT. Moreover, as shown in Fig. 2F, the number of unique TCR clones was significantly associated with the infused cell numbers $\left(r^{2}=0.646, P=0.001\right)$.

\section{Phenotypic analysis of peripheral blood mononuclear cells}

Phenotypic analysis of peripheral blood mononuclear cells prior to the initiation of ACT and at the end of each cycle of adoptive cell therapy was performed. $\mathrm{CD}^{+}$and $\mathrm{CD} 8^{+} / \mathrm{CD} 28^{+} \mathrm{T}$ cell subsets were increased after DC-CIK cell therapy $(\mathrm{P}<$ $0.05)$ (Fig. 4A/B), while the $\mathrm{CD} 4^{+} / \mathrm{CD} 25^{+}$and $\mathrm{CD} 8^{+} / \mathrm{CD} 28^{-}$subsets were significantly decreased after $\mathrm{DC}$-CIK cell therapy $(\mathrm{P}<$ 0.05) (Fig. 4C/D). We performed correlation analysis of changes of T lymphocyte phenotypes with Shannon index and unique TCR subclones as representatives of the diversity of TCR repertoire. The percentages of $C^{2} 3^{+}\left(r^{2}=0.615, P=0.001\right)$ and $\mathrm{CD} 4^{+} / \mathrm{CD} 25^{+} \mathrm{T}$ cells $\left(\mathrm{r}^{2}=0.484, \mathrm{P}=0.001\right)$ were significantly associated with the Shannon index (Fig. 4E); however, the percentages of $\mathrm{CD}^{+} / \mathrm{CD}^{+}\left(r^{2}=0.092, \mathrm{P}=0.222\right)$ and $\mathrm{CD}^{+} / \mathrm{CD} 28^{-}\left(\mathrm{r}^{2}=0.108, \mathrm{P}=0.183\right)$ were not associated with the

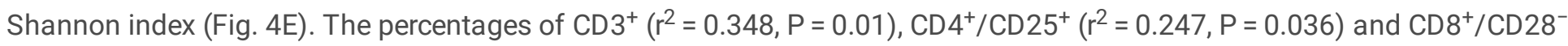


$\left(r^{2}=0.384, P=0.006\right)$ were significantly associated with the unique TCR clones (Fig. 4F); however, the percentage of $\mathrm{CD}^{+} \mathrm{CD}^{+} \mathrm{T}$ cells $\left(\mathrm{r}^{2}=0.022, \mathrm{P}=0.554\right)$ was not correlated with the unique TCR clones (Fig. 4F).

\section{Loss of mutations in ctDNA following DC-CIK infusions}

We explored the ctDNA mutational landscape that occurred during study participation by sequencing ctDNA and gDNA from blood samples obtained at each cycle of infusion. Approximately $10 \mathrm{~Gb}$ and $2 \mathrm{~Gb}$ sequencing data were generated for each ctDNA sample and gDNA sample, respectively. The average depth of coverage was 1323-fold (706-2094) for ctDNA samples. A total of 21 blood samples, collected at an average of 2.5- month intervals, were subjected to complete ctDNA mutational status. A panel of 1021 genes was selected as the target region. Somatic SNVs and InDels were detected using the Mutect 2.0 algorithm

(https://software.broadinstitute.org/gatk/gatkdocs/current/org_broadinstitute_gatk_tools_walkers_cancer_m2_MuTect2.php) Somatic copy number alterations and structure variations were analyzed using local algorithms. Six mutated genes (ARID1B, MYCN, CDH23, SETD2, NOTCH4 and FAT1) were identified in 5/21 samples (Fig. 3). Mutated ARID1B and MYCN disappeared after 3 cycles of $T$ cell infusions and remained absent for 23 months. When the level of $\mathrm{CD}^{+} / \mathrm{CD} 28^{-}$increased to $30.6 \%$ (above the threshold value of $24.2 \%$ ), new missense mutations in SED2 and NOTCH4 were detected. The patient received two cycles of DC/CIK infusion and these mutations disappeared. Subsequently new mutations in the FAT1 gene accompanied an increase in $\mathrm{CD}^{+} / \mathrm{CD} 28^{-}$to $19.6 \%$ which decreased to $15.2 \%$ after T cell infusion (Fig. 3 ) coincident with loss of the mutation in FAT1. A gene mutation of $\mathrm{CDH} 23$, rare in bladder cancer, emerged in the ctDNA when there were elevated $\mathrm{CD} 4^{+} / \mathrm{CD} 25^{+}$ (Treg) but this mutation disappeared from ctDNA after DC-CIK infusion. These data suggest that ctDNA mutation frequency increases with higher immunosuppressive $\mathrm{CD} 8^{+} / \mathrm{CD} 28^{-}$levels and decreases after DC-CIK infusions coincident with decreases in $\mathrm{CD}^{+} / \mathrm{CD} 28^{-}$and $\mathrm{CD} 4^{+} / \mathrm{CD} 25^{+} \mathrm{T}$ cells. These data suggest that during time periods of enhanced activity of immunosuppressive $\mathrm{T}$ cell populations, microscopic residual disease may progress but can be controlled by ACT with DC-CIK infusions.

\section{Discussion}

Immunotherapy has provided new options for treating urothelial carcinomas but responses occur in less than one out of four patients treated with immune checkpoint blockade $[18,19]$, frequently due to a non-inflamed tumor microenvironment. Indeed, in the current case, no expression of either PD-1 or PD-L was identified in tumor biopsies. Studying an alternative form of immunotherapy, we have previously reported that infusions of mixtures of dendritic cells and cytokine induced killer cells (DC-CIK) demonstrated efficacy in several solid tumor malignancies [9, 10, 14, 20-22]. We designed an N-of-1 study to characterize the changes in immune cell profiles of a patient with NMIBC who refused radical cystectomy and instead opted to participate in a study of immunotherapy with DC-CIK after completing standard interventions. Peripheral blood samples were serially collected during their 6 years of treatment. This allowed us to correlate immune cell alterations with standard clinical imaging results. Specifically, we had the opportunity to perform a detailed analysis of T cell phenotype and receptor repertoire from multiple samples from the same patient and studied their relationship to the number of cells infused. Simultaneously, we incorporated circulating tumor DNA mutation analysis to explore whether tumor progression would be detectable at the molecular level during times when suppressor T cell populations were highest.

A unique aspect of this case was the use of peripheral blood $\mathrm{CD} 8^{+} / \mathrm{CD} 28^{-}$and $\mathrm{CD} 4^{+} / \mathrm{CD} 25^{+} / \mathrm{CD} 127^{+} \mathrm{T}$ cells as an indicator for timing of DC-CIK infusions. $\mathrm{CD} 4^{+} / \mathrm{CD} 25^{+} \mathrm{T}$ cells include populations of regulatory $\mathrm{T}$ cells. Accumulating evidence indicates that $\mathrm{CD} 8^{+} / \mathrm{CD} 28^{-} \mathrm{T}$ cells are associated with numerous inflammatory disorders and are found in tumor microenvironments and the circulation of cancer patients. Previously, we observed that increases in these suppressor cell types were associated with worse outcome in breast cancer patients and were predictive of cancer cell release into the blood stream [23]. In the current study, when suppressor cell subtypes increased, circulating tumor DNA mutations (in genes with relevance for tumor aggressiveness[24-27]) appeared and after delivery of DC-CIK, these suppressor cells decreased and the 
mutations disappeared. This suggests that the DC-CIK were responsible for sustaining the complete remission by modulating suppressor populations and/or by direct anti-tumor effects.

During the continuous sample analysis for ctDNA mutation, we have observed that there were six tumor associated gene mutations were recorded. We have compared these six genes with gene database to explain the mutational results. As the tumor suppressor genes as ARID1B, the loss of function could give rise to tumor progressive behavior, the histopathological study reported by Sano et al to demonstrate that low expression of ARID1B among the ovarian clear cell carcinoma patients was associated with shorten progression free survival rate[24]. We have found that such gene mutational frequency was dramatically reduced when the T cell infusion executed. When the level of $\mathrm{CD} 8^{+} / \mathrm{CD} 28^{-}$increased to $30.6 \%$ (above the threshold value of $24.2 \%$ ), new missense mutations in SED2 and NOTCH4 were detected. The patient received two cycles of $\mathrm{DC} / \mathrm{CIK}$ infusions and these mutations disappeared. We have some conviction data to support the phenomenon that $\mathrm{T}$ suppressor cells elevated from the peripheral blood was predictor to clinical tumor progression, little knew that occurrence of SETD2 and NOTCH4 mutation were risen from tumor progression or accompanying transit events followed by DC/CIK immunotherapy as some publications have revelations SETD2, MYCN and NOTCH4 could induced drug resistance and initiation of cancer progression[25,26].. Nether less the subsequently new mutations in the FAT1 gene accompanied an increase in $\mathrm{CD}^{+} / \mathrm{CD} 28^{-}$to $19.6 \%$ which decreased to $15.2 \%$ after T cell infusion (Fig. 3 ) coincident with loss of the mutation in FAT1. In particular we have noticed that the rare gene mutation in $\mathrm{CDH} 23$, which has been implied on the evidence that $\mathrm{CDH} 23$ expression was down-regulated DNA methylation of lung and esophageal cancer, suppressing tumor metastasis and cell migration.[27] The loss of $\mathrm{CDH} 23$ mutation was presumably associated with $\mathrm{T}$ cell infusion in this patient along with the improvement of T cell phenotype.

Our finding that there was a significant correlation between suppressive T cell populations and TCR repertoire profiles (such as Shannon index and unique T cell clonality) (as shown in Fig. 4, E,F) supports the former mechanism, in that the effector T cell repertoire may be too limited to control tumor when there are greater levels of suppressor cell subtypes present. Further, our finding that the TCR diversity and unique TCR clones in peripheral blood after DC-CIK administration were significantly associated with the number of infused cells[20] suggests that DC-CIK can provide at least a temporary supply of additional anti-tumor T cells. This is consistent with our previous report that T cell immunotherapy is able to restore the TCR repertoire with increased unique $T$ cell clones which could enhance the specific $T$ cell immune response to tumor antigens.

\section{Conclusions}

Based on our experience, we hypothesize that a threshold of suppressor cells (above which new mutations in ctDNA were observed) can be identified that could serve as a criterion for the timing of T cell immunotherapy; however, because this report consists of data from a single patient, a larger study would be required to conclusively determine whether infusions of $T$ cells at routine intervals or in reaction to waning immunity (or detection of new or increasing frequency of mutations in ctDNA) would be the more effective strategy.

\section{Abbreviations}

NMIBC: non-muscle-invasive bladder cancer; ACT: activated T cell immunotherapy; DC: dendritic cell; CIK: cytokine-induced killer cell; TCR: T cell receptor; ctDNA: circulating tumor DNA; NGS: next generation sequencing; TURBT: transurethral resection; BCG: bacillus Calmette-Guerin; HTTCR:high-throughput T cell receptor V $\beta$ sequencing; SD: standard deviation; PBMC: Peripheral blood mononuclear cell

\section{Declarations}

Ethics approval and consent to participate 
The treatment for this patient was performed in accordance with Declaration of Helsinki after receiving approval from the Institutional Review Board of Beijing Shijitan Hospital and with informed consent.

\section{Consent for publication}

Written informed consent was obtained from the patient.

\section{Availability of data and material}

The datasets supporting the conclusions are included in this published article. The datasets used and/or analyzed during the current study are available from the corresponding author on reasonable request.

\section{Competing interests}

The authors declare that they have no competing interests.

\section{Funding}

This work was supported by Key Project of Beijing Municipal Committee of Science and Technology-Capital Clinical Featured Application Funding (Z151100004015183); National Natural Science Foundation of China (81602528); Enhancement Funding of Laboratory of Beijing Key Laboratory for Therapeutic Cancer Vaccines (2020-JS01).

\section{Authors' contributions}

JR and HKL who supervised the research, conducted the study and had full access to all data in the study and took responsibility for the integrity of the data and the data accuracy. JR was a major contributor in writing the manuscript and obtained the funding. XLW participated in the clinical care, acquisition of data, analysis and interpretation of data, administrative and a major contributor in writing the manuscript. GLQ was a major contributor in statistical analysis the data, writing the manuscript and obtaining funding. NJ participated in the clinical care, acquisition of data冈interpretation of data and material support. MAM participated in study concept and critical revision of the manuscript for important intellectual content. SW,YGS, YJZ , LZ participated in the clinical care. JPW performed flow cytometry. XNZ participated in acquisition of data and material support.. YHY produced clinical cell products. HKL, AH participated in critical revision of the manuscript for important intellectual content. All authors read and approved the final manuscript

\section{Acknowledgements}

We would like to acknowledge the expert technical assistance of Xuefeng Xia and Xin Yi Ph.D. from Geneplus-Beijing Institute, Beijing China.

\section{References}

1. Torre LA, Bray F, Siegel RL, Ferlay J, Lortet-Tieulent J, Jemal A. Global cancer statistics, 2012. CA Cancer J Clin. 2015;65(2):87-108.

2. Cookson MS, Herr HW, Zhang ZF, Soloway S, Sogani PC, Fair WR. The treated natural history of high risk superficial bladder cancer: 15-year outcome. The Journal of urology. 1997;158(1):62-7.

3. Shen PL, Lin ME, Hong YK, He XJ. Bladder preservation approach versus radical cystectomy for high-grade non-muscleinvasive bladder cancer: a meta-analysis of cohort studies. World J Surg Oncol. 2018;16(1):197.

4. Necchi A, Briganti A, Bianchi M, Raggi D, Giannatempo P, Freschi M, Colecchia M, Fossati N, Gandaglia G, Colombo R: Abstract CT003: Preoperative pembrolizumab (pembro) before radical cystectomy (RC) for muscle-invasive urothelial bladder carcinoma (MIUC): Interim clinical and biomarker findings from the phase II PURE-01 study. In.: AACR; 2018. 
5. Study of Pembrolizumab (MK-3475) in Participants With High Risk Non-muscle Invasive Bladder Cancer (MK-3475057/KEYNOTE-057). https://clinicaltrials.gov/ct2/show/NCT02625961. Accessed 2 June, 2019.

6. Sweis RF, Spranger S, Bao R, Paner GP, Stadler WM, Steinberg G, Gajewski TF. Molecular Drivers of the Non-T-cellInflamed Tumor Microenvironment in Urothelial Bladder Cancer. Cancer immunology research. 2016;4(7):563-8.

7. Li W, Xu LP, Wang LDIZ, Zhang L, Gao Y, Mai QL. L: Cytokine-induced killer cell therapy for advanced pancreatic adenocarcinoma: A case report and review of the literature. Oncol Lett. 2013;5(4):1427-9.

8. Chung MJ, Park JY, Bang S, Park SW, Song SY. Phase II clinical trial of ex vivo-expanded cytokine-induced killer cells therapy in advanced pancreatic cancer. Cancer Immunol Immunother. 2014;63(9):939-46.

9. Jiang N, Qiao G, Wang X, Morse MA, Gwin WR, Zhou L, Song Y, Zhao Y, Chen F, Zhou X, et al. Dendritic Cell/CytokineInduced Killer Cell Immunotherapy Combined with S-1 in Patients with Advanced Pancreatic Cancer: A Prospective Study. Clin Cancer Res. 2017;23(17):5066-73.

10. Qiao G, Wang X, Zhou L, Zhou X, Song Y, Wang S, Zhao L, Morse MA, Hobeika A, Song J, et al. Autologous Dendritic CellCytokine Induced Killer Cell Immunotherapy Combined with S-1 Plus Cisplatin in Patients with Advanced Gastric Cancer: A Prospective Study. Clin Cancer Res. 2019;25(5):1494-504.

11. Robins HS, Campregher PV, Srivastava SK, Wacher A, Turtle CJ, Kahsai O, Riddell SR, Warren EH, Carlson CS. Comprehensive assessment of T-cell receptor beta-chain diversity in alphabeta T cells. Blood. 2009;114(19):4099-107.

12. Robert L, Tsoi J, Wang X, Emerson R, Homet B, Chodon T, Mok S, Huang RR, Cochran AJ, Comin-Anduix B, et al. CTLA4 blockade broadens the peripheral T-cell receptor repertoire. Clinical cancer research: an official journal of the American Association for Cancer Research. 2014;20(9):2424-32.

13. Gros A, Parkhurst MR, Tran E, Pasetto A, Robbins PF, llyas S, Prickett TD, Gartner JJ, Crystal JS, Roberts IM, et al. Prospective identification of neoantigen-specific lymphocytes in the peripheral blood of melanoma patients. Nature medicine. 2016;22(4):433-8.

14. Ren J, Di L, Song G, Yu J, Jia J, Zhu Y, Yan Y, Jiang H, Liang X, Che L, et al. Selections of appropriate regimen of highdose chemotherapy combined with adoptive cellular therapy with dendritic and cytokine-induced killer cells improved progression-free and overall survival in patients with metastatic breast cancer: reargument of such contentious therapeutic preferences. Clin Transl Oncol. 2013;15(10):780-8.

15. Rempala GA, Seweryn M. Methods for diversity and overlap analysis in T-cell receptor populations. J Math Biol. 2013;67(6-7):1339-68.

16. Qi Q, Liu Y, Cheng Y, Glanville J, Zhang D, Lee JY, Olshen RA, Weyand CM, Boyd SD, Goronzy JJ. Diversity and clonal selection in the human T-cell repertoire. Proc Natl Acad Sci USA. 2014;111(36):13139-44.

17. Kvalseth TO. Evenness indices once again: critical analysis of properties. SpringerPlus. 2015;4:232.

18. Faiena I, Cummings AL, Crosetti AM, Pantuck AJ, Chamie K, Drakaki A. Durvalumab: an investigational anti-PD-L1 monoclonal antibody for the treatment of urothelial carcinoma. Drug Des Devel Ther. 2018;12:209-15.

19. Sweis RF, Zha Y, Pass L, Heiss B, Chongsuwat T, Luke JJ, Gajewski TF, Szmulewitz R. Pseudoprogression manifesting as recurrent ascites with anti-PD-1 immunotherapy in urothelial bladder cancer. J immunother Cancer. 2018;6(1):24.

20. Ren J, Gwin WR, Zhou X, Wang X, Huang H, Jiang N, Zhou L, Agarwal P, Hobeika A, Crosby E, et al. Adaptive T cell responses induced by oncolytic Herpes Simplex Virus-granulocyte macrophage-colony-stimulating factor therapy expanded by dendritic cell and cytokine-induced killer cell adoptive therapy. Oncoimmunology. 2017;6(4):e1264563.

21. Wang X, Ren J, Zhang J, Yan Y, Jiang N, Yu J, Di L, Song G, Che L, Jia J, et al. Prospective study of cyclophosphamide, thiotepa, carboplatin combined with adoptive DC-CIK followed by metronomic cyclophosphamide therapy as salvage treatment for triple negative metastatic breast cancers patients (aged < 45). Clin Transl Oncol. 2016;18(1):82-7.

22. Zhao Y, Qiao G, Wang X, Song Y, Zhou X, Jiang N, Zhou L, Huang H, Zhao J, Morse MA, et al. Combination of DC/CIK adoptive $T$ cell immunotherapy with chemotherapy in advanced non-small-cell lung cancer (NSCLC) patients: a prospective patients' preference-based study (PPPS). Clin Transl Oncol. 2019;21(6):721-8. 
23. Song Q, Ren J, Zhou X, Wang X, Song G, Hobeika A, Yuan Y, Lyerly HK. Circulating CD8(+)CD28(-) suppressor T cells tied to poorer prognosis among metastatic breast cancer patients receiving adoptive T-cell therapy: A cohort study. Cytotherapy. 2018;20(1):126-33.

24. Sato E, Nakayama K, Razia S, Nakamura K, Ishikawa M, Minamoto T, Ishibashi T, Yamashita H, lida K, Kyo S. ARID1B as a Potential Therapeutic Target for ARID1A-Mutant Ovarian Clear Cell Carcinoma. Int J Mol Sci 2018, 19(6).

25. Wang S, Yuan X, Liu Y, Zhu K, Chen P, Yan H, Zhang D, Li X, Zeng H, Zhao X, et al. Genetic polymorphisms of histone methyltransferase SETD2 predicts prognosis and chemotherapy response in Chinese acute myeloid leukemia patients. Journal of translational medicine. 2019;17(1):101.

26. Montemurro L, Raieli S, Angelucci S, Bartolucci D, Amadesi C, Lampis S, Scardovi AL, Venturelli L, Nieddu G, Cerisoli L, et al. A Novel MYCN-Specific Antigene Oligonucleotide Deregulates Mitochondria and Inhibits Tumor Growth in MYCNAmplified Neuroblastoma. Cancer research. 2019;79(24):6166-77.

27. Sannigrahi MK, Srinivas CS, Deokate N, Rakshit S. The strong propensity of Cadherin-23 for aggregation inhibits cell migration. Mol Oncol. 2019;13(5):1092-109.

\section{Figures}


A

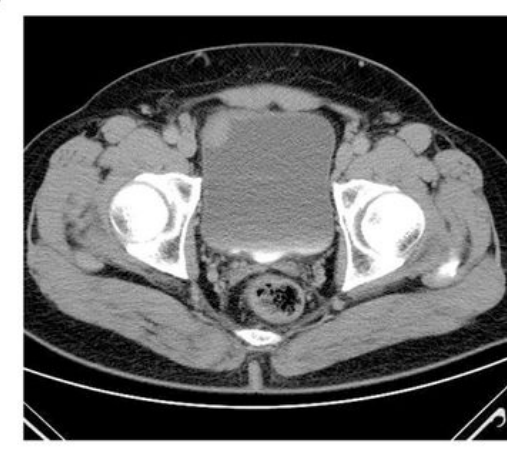

D

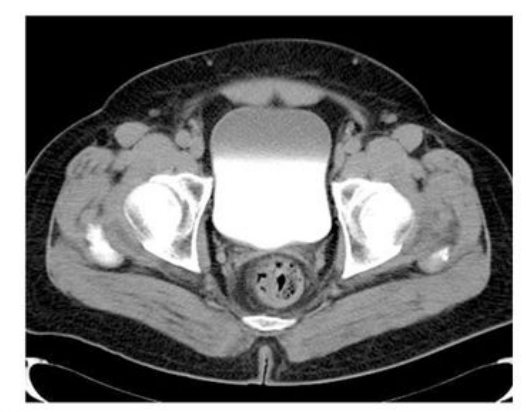

G

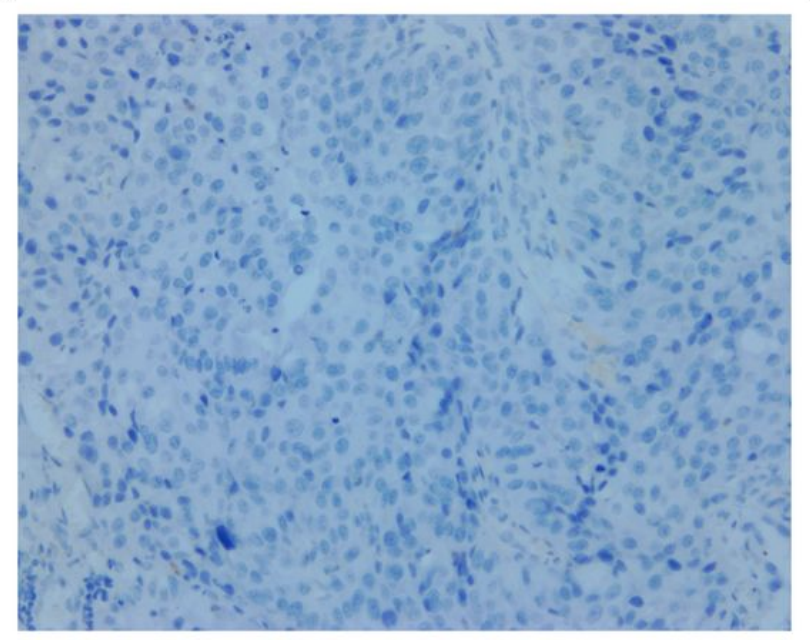

C
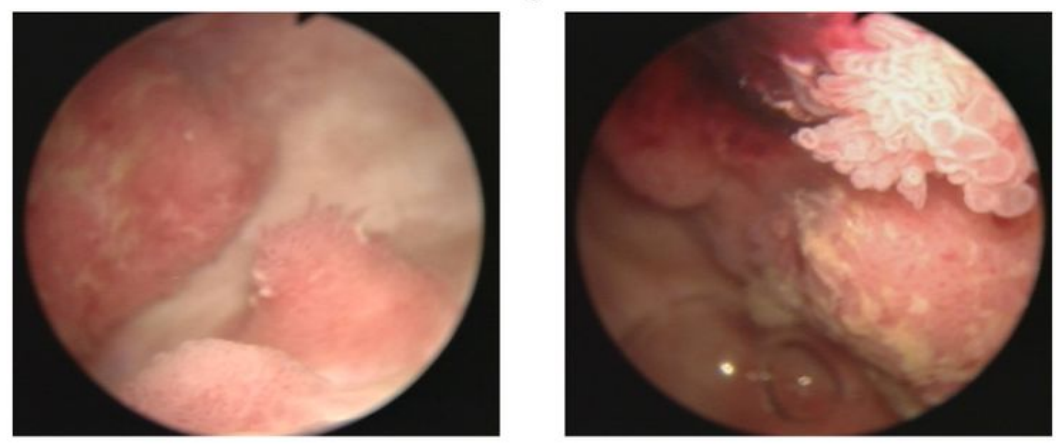

$\mathbf{F}$

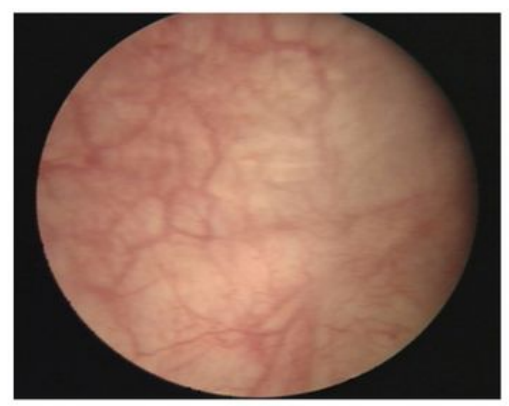

H

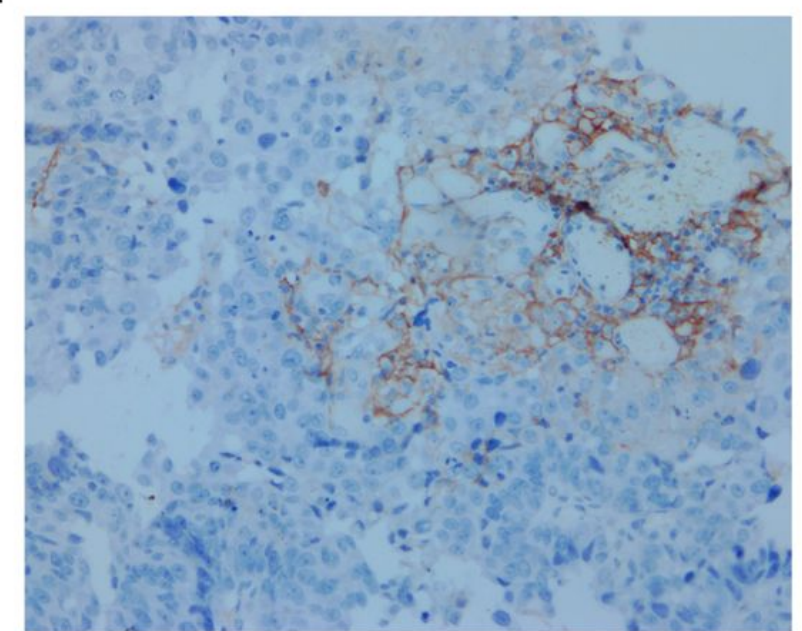

\section{Figure 1}

Pelvic CTs and cystoscopies before (A-C) and after (D-F) treatment with DC-CIK therapy; Immunohistochemical analysis showed that tumor tissue prior to therapy demonstrated no PD-1 (G) or PD-L1 (H) expression 

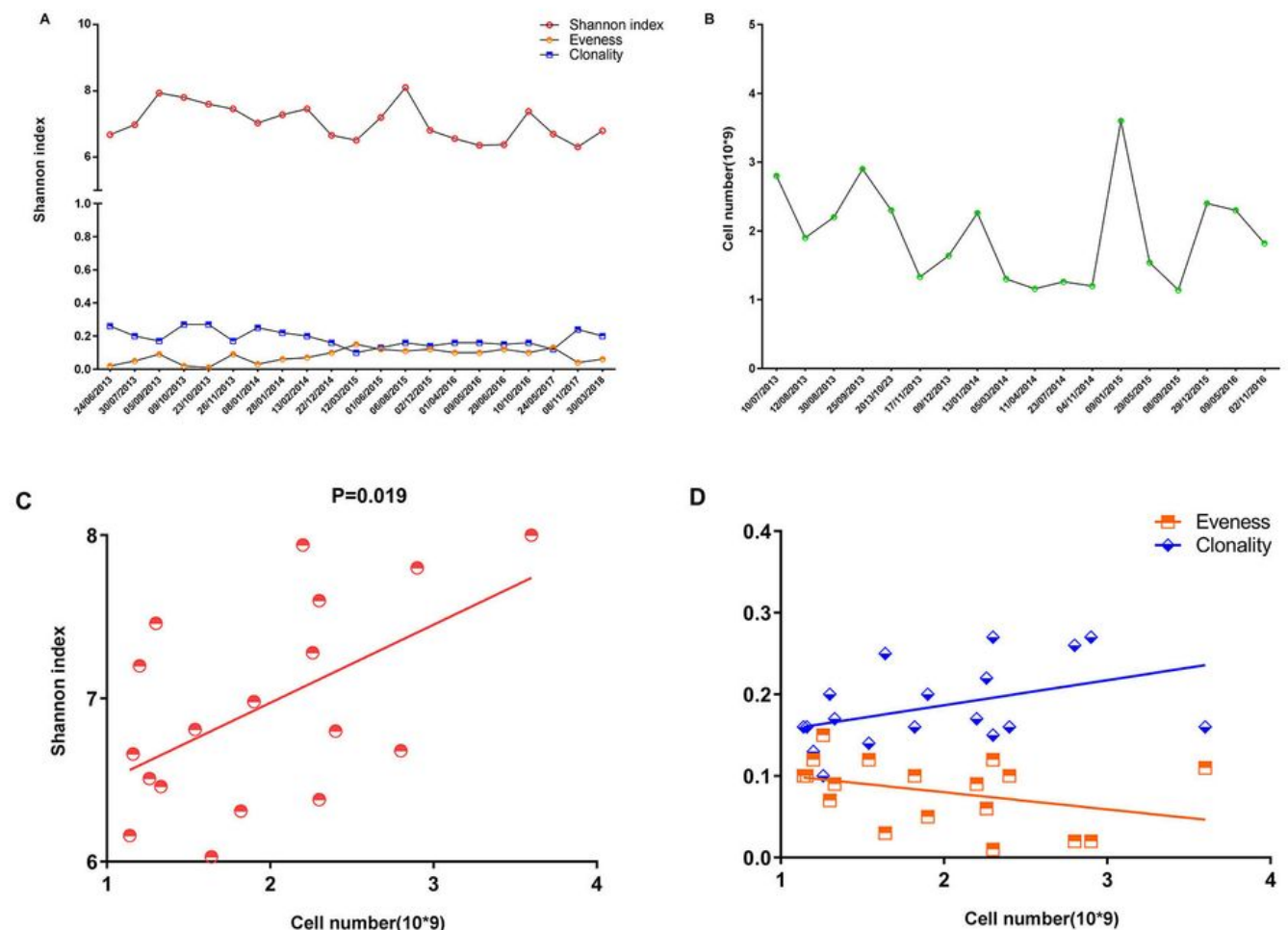

D

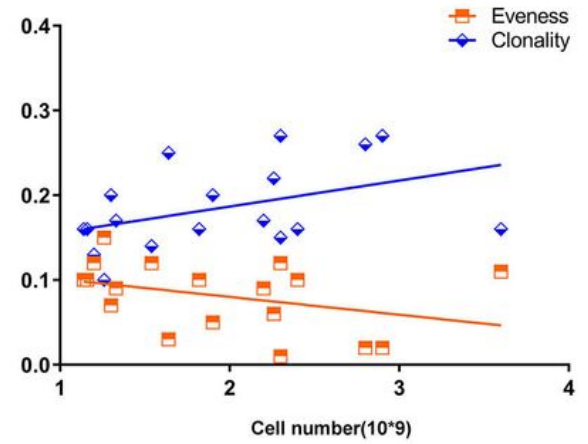

$E$

T cell clones
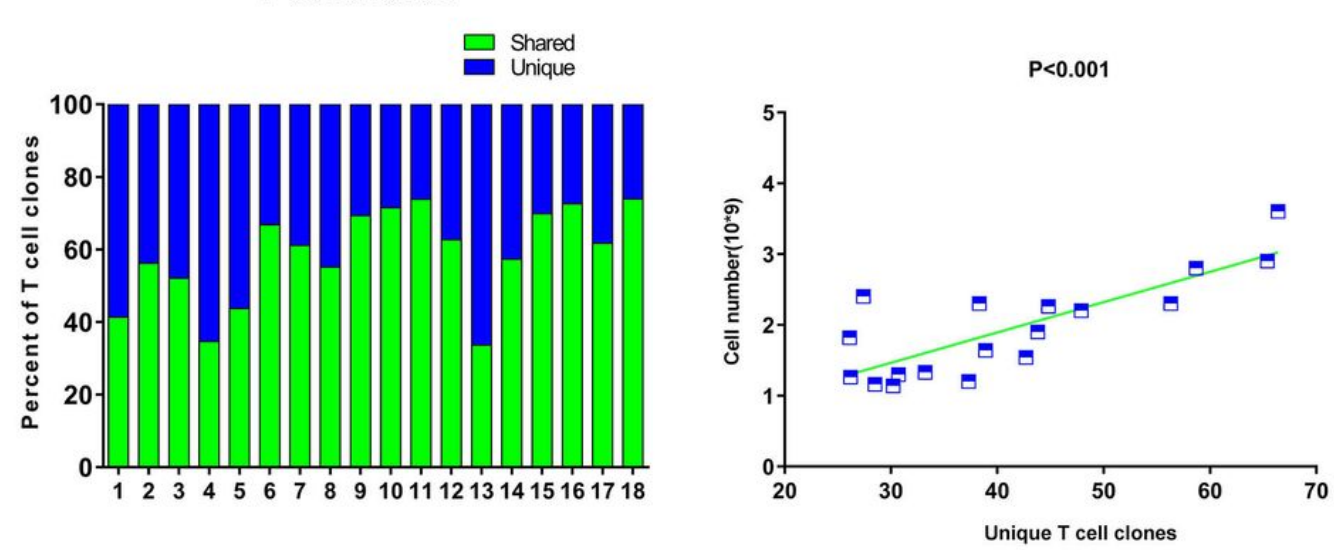

Figure 2

TCR diversity after DC-CIK therapy is associated with the infused cell number. A,B: Alteration of Shannon index and infused cell number are shown; C: The Shannon index was significantly associated with the infused cell number; D: The clonality and eveness were not significantly associated with the infused cell number; E: Changes in TCR subclones after DC-CIK therapy; F: The association of unique TCR clones with infused cell number. 


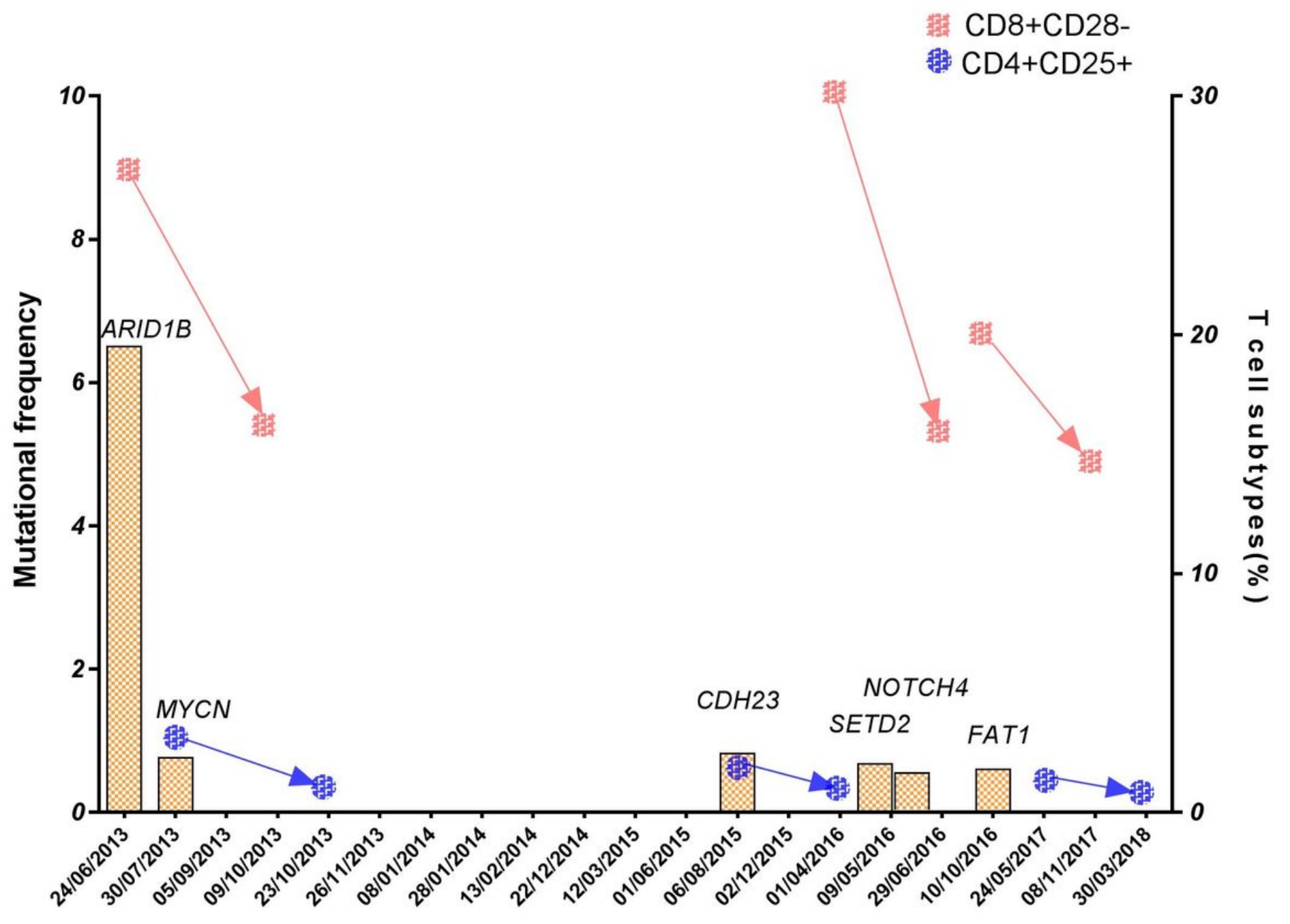

Figure 3

ctDNA mutational analysis and changes in CD4+/CD25+ and CD8+/CD28- T cell proportions during the study participation. 

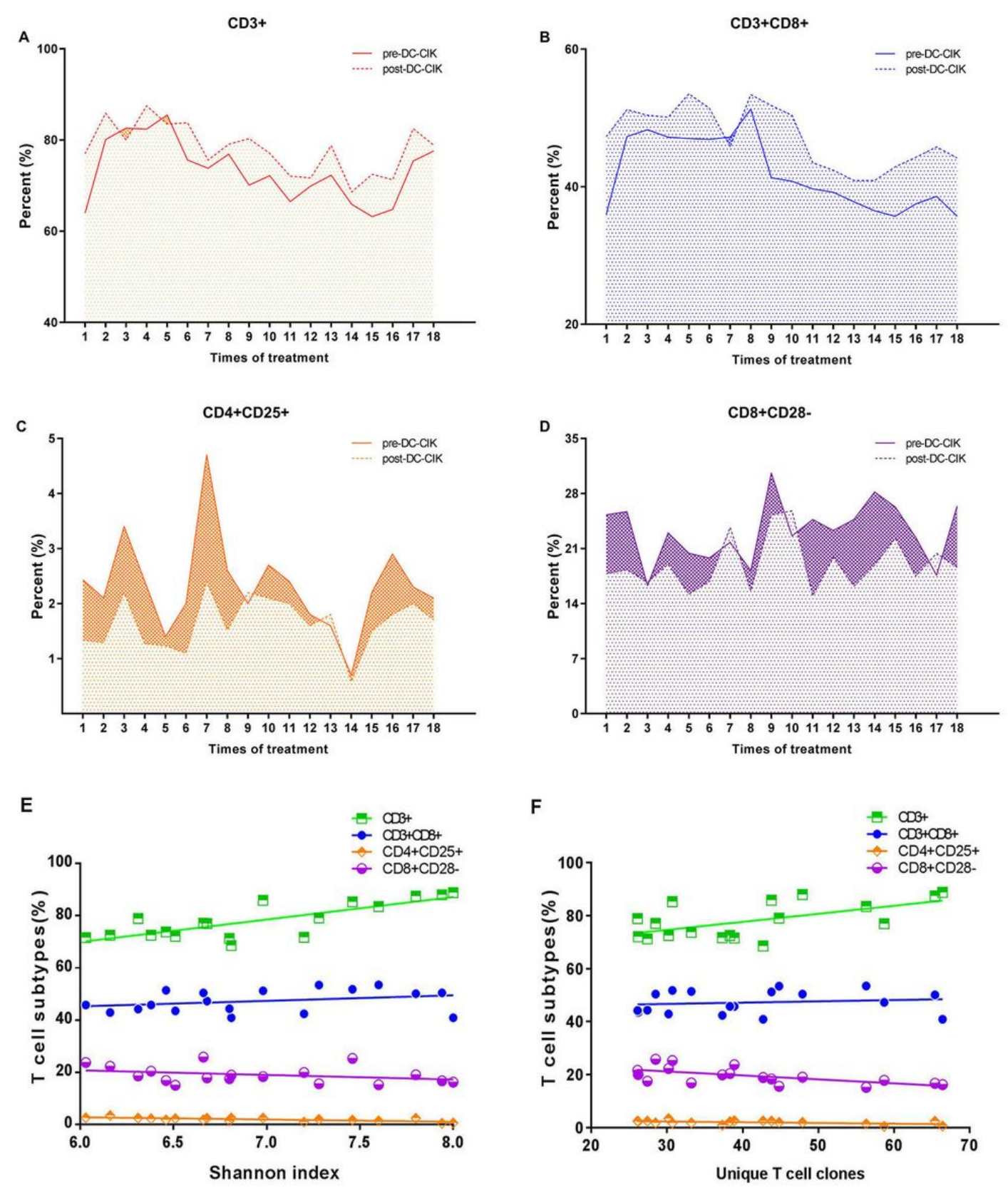

\section{Figure 4}

T lymphocyte subsets in peripheral blood are shown before and after DC-CIK therapy. A-D: Peripheral blood T cell phenotype characterized by flow cytometry before and after the infusions of DC-CIK therapy. The subtypes of CD3+, CD3+/CD8+, CD4+/CD25+ and CD8+/CD28- T cells showed significant differences; E: The association of T cell phenotype with Shannon index; F: The association of T cell phenotype with unique T cell clones. 